\title{
Article
}

\section{Rescheduling Plan Optimization of Underground Mine Haul- age Equipment Based on Random Breakdown Simulation}

\author{
Ning LI ${ }^{1,2,}$, , Shuzhao FENG $1{ }^{, *}$, Tao LEI $1{ }^{1,{ }^{*},}$, Haiwang YE, Qizhou WANG, Liguan WANG ${ }^{3}$, Mingtao JIA ${ }^{3}$ \\ 1 School of Resource and Environment Engineering, Wuhan University of Technology, Wuhan, Hubei 430070, \\ China; fsz2224284697@163.com (S.F.); leitao539@163.com (T.L.); yehaiwang@sina.com (H.Y.); \\ wqz@whut.edu.cn (Q.W.); \\ 2 Hubei Key Laboratory of Mineral Resources Processing and Environment, Wuhan Hubei 430070, China; \\ 3 School of Resource and Safety Engineering, Central South University, Changsha Hunan 410083, China; \\ 13808478410@163.com (L.W.); mingtao jia@163.com (M.J.); \\ * Correspondence: 13875910191@163.com (N.L.); fsz2224284697@163.com (S.F.); leitao539@163.com (T.L.); \\ Tel.: +86-138-7591-0191
}

\begin{abstract}
Due to production space and operating environment requirements, mine production equipment often breaks down, which seriously affects the mine's production schedule. To ensure the smooth completion of the haulage operation plan under abnormal conditions, a model of the haulage equipment rescheduling plan based on the random simulation of equipment breakdowns is established in this paper. The model aims to accomplish both the maximum completion rate of the original mining plan and the minimum fluctuation of the ore grade during the rescheduling period. This model is optimized by improving the wolf colony algorithm and changing the location update formula of the individuals in the wolf colony. Then, the optimal model solution can be used to optimize the rescheduling of the haulage plan by considering equipment breakdowns. The application of the proposed method in an underground mine revealed that the completion rate of the mine's daily mining plan reached $83.40 \%$ without increasing the number of the equipment, while and the ore quality was stable. Moreover, the improved optimization algorithm converged fast and was characterized by high robustness.
\end{abstract}

Keywords: project scheduling; underground mine; random breakdown simulation; wolf colony algorithm; multi-objective optimization

\section{Introduction}

To reduce the waste of various resources and indirectly protect the environment, the proposal of the concept of the green mine requires a scientific mining process and efficient utilization of equipment resources. Standardized and intensive mining are the main mining modes of the future. Underground production equipment is gradually developing in a large-scale and intelligent direction. Therefore, effectively improving the mining efficiency and equipment utilization will become one of the key parameters for developing green mines and improving the economic benefits of mining enterprises. With the continuous development of information technology, big data, and artificial intelligence, intelligent mining theory and technology have come a long way, thereby providing basic support for the transformation of mining enterprises from extensive to refined. Optimal preparation of production plan and reasonable equipment scheduling are important aspects of green mines and intelligent mining. However, due to factors such as the mining environment, equipment performance, and personnel quality, abnormal conditions such as equipment breakdowns and safety accidents will inevitably occur during the production process. As a result, the original scheduling plan may not be completed, which in turn affects the overall production schedule of the mine and the economic benefits of the en- 
terprise. Therefore, solving the problem of production equipment rescheduling under abnormal conditions and completing the original production plan to the greatest extent have become new research hotspots in the field of green mines and intelligent mining.

Regarding the preparation of underground mine production plans, Yao et al. established a mathematical model of underground mine ore allocation. Moreover, the authors employed the immune cloning algorithm to solve the model and prove its feasibility [1]. To achieve dynamic and scientific validity of the production plan preparation for polymetallic underground mines and maximize profits, Hou et al. established the dynamic optimization model of production plan based on technical and economic requirements as well as spatial sequence relationship in the mining process. The proposed model improves the economic benefits while ensuring the continuity of production [2]. Campeau \& Gamache proposed an optimization model for short-term planning. The model considers all parts of development and production, as well as specific restrictions on equipment and workers. Moreover, the model employs a preemptive mixed-integer program to prepare the best production planning in a short time frame [3].

Regarding the production equipment scheduling plan and scientifically achieving the compactness of underground mine production and schedule production equipment, many investigations can also be found in the literature. Li et al. established an integrated optimization model for underground mine production continuity and equipment scheduling with the shortest process interval as well as the shortest total completion time. While ensuring the completion of the specified tasks, the proposed model effectively shortens the process time interval and ensures the safety requirements of the production [4]. To reduce the ineffective travel time of haulage equipment, Yardimci \& Karpuz proposed a shortest path optimization method for underground mine haulage road design based on the evolutionary algorithm. This method considers kinematic constraints such as minimum turning radius and maximum slope to determine the optimal haulage path [5]. For such instances, it is necessary to prepare high-quality operation planning for haulage equipment. Åstrand et al. proposed a constraint programming method that can automatically execute short-term scheduling plans, employ large-scale neighborhood search with neighborhood definitions in specific areas, and contribute to finding high-quality schedules faster [6].

The aforementioned studies assume that the equipment keeps normal operation during the production period, i.e., they do not consider the breakdown of the equipment and similar situations. Samatemba et al. used Rstudio software to develop an algorithm and evaluate sensitive inputs of the life cycle of mining equipment. Moreover, the algorithm was used to determine, analyze, and optimize the overall equipment efficiency [7]. After the equipment performance of the haulage equipment was evaluated, the breakdown probability of the equipment was calculated by counting the number of breakdowns and constructing a random breakdown algorithm to simulate the moment of breakdown. Zandieh \& Gholami investigated the mixed flow shop scheduling problem with sequencerelated setup time and random machine breakdowns. The authors employed the expected completion time as the optimization goal [8]. Al-Hinai \& Elmekkawy solved the problem of flexible job shop scheduling with random breakdowns. Based on opportunity-constrained programming [9]. Ai et al. comprehensively considered random factors such as unit breakdowns, line breakdowns, load forecast errors, and interruptible load defaults. Moreover, the authors took the minimum system operating cost as the objective function to construct an optimization model with interruptible loads [10]. Based on the internal relationship between the scheduling scheme structure, machine breakdown probability, maintenance time, and scheduling robustness, Ba et al. proposed an alternative measurement method based on the propagation of process breakdown effects. The authors wanted to investigate the problem of measuring the robustness of job shop scheduling in a random machine breakdown environment [11].

Researchers have made considerable progress in rescheduling. The main research work can be carried out in three steps. Firstly, the breakdown problem is considered [12], 
and the original plan is reprogrammed. Secondly, the existing rescheduling model is employed to construct a new rescheduling model [13], or corresponding rescheduling strategies [14]-[18] and frameworks [19] are proposed according to different breakdown problems. When proposing the rescheduling strategy, some researchers proposed that a method can be used to determine whether the rescheduling is required [20]. Finally, based on completing the construction of the rescheduling model, the corresponding optimization algorithm [21]-[24] is improved to solve the strategy model.

In this paper, an optimization method of underground mine haulage equipment rescheduling plan based on random breakdowns simulation is proposed. The main tasks of the method are:

(1) Constructing a random breakdown algorithm, determining the time of breakdown occurrence and maintenance end, and classifying and segmenting those times according to whether each breakdown time is intersected or not.

(2) Constructing a rescheduling plan model during each equipment breakdown period. Here, the main goal of minimum ore grade deviation and the secondary goal of maximum completion rate of the planning during the rescheduling period are taken. Finally, the rescheduling plan model is solved via the improved wolf pack algorithm.

(3) Comparing the solution results of the rescheduling plan optimization model with the traditional scheduling plan to verify its feasibility.

\section{Model}

\subsection{Prerequisites and problem description}

\subsubsection{Prerequisites}

By considering the adjustment strategy for equipment breakdowns in the actual mining process, the following assumptions are made:

(1) When the haulage equipment breaks down, there is no spare equipment for dispatching, and the malfunctioning equipment can only continue to operate after the repair is completed.

(2) During the daily planning period, the haulage equipment can only be operated in a single sublevel.

(3) When the equipment breaks down, to avoid affecting the haulage of the line, auxiliary equipment is used to relocate it to a free area for maintenance.

(4) After the equipment maintenance is completed, the start time of the equipment's continued operation is designated according to the time required to finish this operation.

(5) During the daily planning period, each piece of equipment breaks down at most once.

\subsubsection{Problem description}

When the mine adopts the caving method for mining, it mainly relies on scrapers and electric locomotives for ore haulage. The task of the scraper is to transport the ore from the stope to the ore pass in a sublevel. The electric locomotive is responsible for transporting the ore from the ore pass to the main shaft.

Since the electric locomotive runs relatively slowly and travels on a fixed and smooth track, its probability of breakdown is relatively low. Compared with electric locomotives, the working environment of scrapers is more complicated. Humid air and bumpy roads increase the breakdown probability of the equipment. In addition, long-term turns and gear changes may cause fatigue and deformation of the machine parts, thereby increasing the risk of a potential breakdown.

According to the breakdown repair time of each piece of equipment, two rescheduling strategies are proposed: inserting rescheduling strategy and complete rescheduling strategy. For the former, when a piece of certain equipment breaks down, the original 
scheduling plan in this period is re-prepared during the maintenance period of the equipment. For the latter, when a piece of certain equipment breaks down, all subsequent uncompleted original scheduling plans are re-prepared during the maintenance period of the equipment. Consequently, all subsequent uncompleted original scheduling plans are still re-prepared after the equipment maintenance is completed.

When the breakdown repair time of each piece of equipment intersects on the timeline, the breakdown time is divided into multiple rescheduling periods. This is done by taking the crossing point between the breakdown time of each piece of equipment and the time when the breakdown occurs and maintenance ends. Then, the inserting rescheduling plan preparation is implemented within each rescheduling period. When the breakdown repair time of each piece of equipment does not intersect on the timeline, all subsequent periods are divided into multiple complete rescheduling periods starting from the moment of the first breakdown. This is done when the equipment is characterized by the first breakdown within the daily planning period. In such instances, the complete rescheduling plan is prepared within each rescheduling period (Figure 1).

$\# 1$

$\# 2$

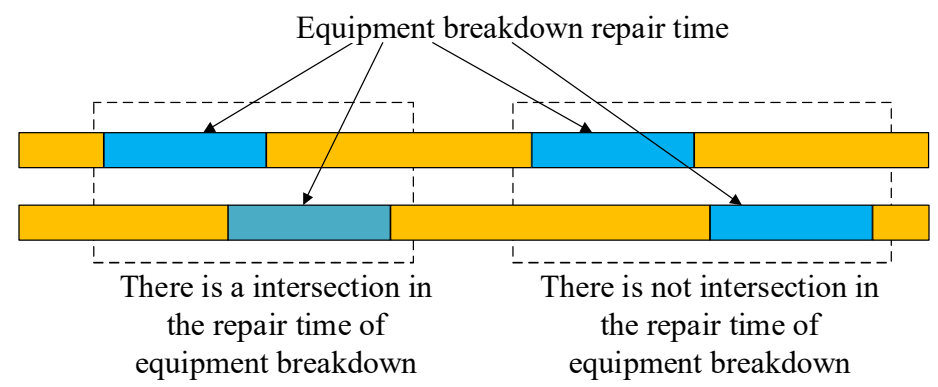

Figure 1. The intersection of each equipment breakdown repair time on the timeline.

The problem of rescheduling haulage equipment is described as follows. The mine point is denoted as $C_{i}$ and the dump point as $X_{\mathrm{j}}$. The haulage equipment $M_{\mathrm{k}}$ runs between the mine point and the dump point, $i=1,2, \ldots, m, j=1,2, \ldots, n, k=1,2, \ldots, l$. Parameter $G_{\mathrm{k}}$ represents the moment when the $k$-th equipment fails. $D_{\mathrm{kij}}$ and $D_{\mathrm{kji}}$ represent that the $k$-th equipment is located between the $i$ mine point and the $j$ dump point, respectively representing $i \rightarrow j$ and $j \rightarrow i$. Lastly, $W_{\mathrm{k}}$ denotes the maintenance time of the $k$-th equipment. The preparation of the rescheduling plan is done by rescheduling the rest of the normal operating equipment within the specific time range. To meet the original scheduling task as much as possible, this time range is defined from the breakdown of each piece of equipment to the end of the maintenance.

The rescheduling plan cycle is defined as a single day, while the time period is defined as 30 minutes. The probability of breakdown of the haulage equipment at each time period is statistically analyzed. The random breakdown algorithm is described as follows:

(1) Divide the single day into 48 parts, $t \in[1,48], t=1$.

(2) Choose a random number $R_{\mathrm{tk}}$ for each period which represents the random number for the $t$-th period of the $k$-th equipment.

(3) Compare this random number with the breakdown probability $P_{\text {tk }}$ of the $k$-th equipment in the $t$-th period. If $R_{\mathrm{tk}}>P_{\mathrm{tk}}$, then the $k$-th equipment does not break down in the $t$-th period. Otherwise, it is considered that a breakdown has occurred.

(4) The specific breakdown time point is randomly obtained from the planning within the $t$-th period.

(5) When a breakdown occurs in the $t$-th period, the repair end time is $W_{\mathrm{k}}+0.5 t$. Then, the $t^{\prime}$-th period, which is the repair end period, is determined.

(6) The $t$ starts from $t^{\prime}+1$, and step (2) is repeated. Finally, the occurrence times for all breakdowns are determined and the end of maintenance of each equipment within the daily planning period. 


\subsection{Model building}

A strategy of inserting a rescheduling plan is proposed to consider simultaneously repairing two or more pieces of equipment. For such instances, simultaneous breakdown of multiple pieces of equipment will seriously affect the execution of the original planning. Therefore, it is necessary to re-prepare the unfinished original planning during the equipment repair period.

If the breakdown repair time of each piece of equipment does not intersect, i.e., there is only one piece of equipment that is being repaired, the impact during the repair period is smaller. Therefore, a complete rescheduling plan strategy is proposed to re-prepare subsequent uncompleted original planned tasks.

\subsubsection{Sets}

J: Set of stopes that participated in the original planning.

$$
\mathcal{J}=\{1, \ldots, I\}, i \in \mathcal{J}
$$

J: Set of ore passes that participated in the original planning.

$$
\mathcal{J}=\{1, \ldots, J\}, j \in \mathcal{J}
$$

$\mathcal{M}$ : Equipment sets that participated in the original planning.

$$
\mathcal{M}=\{1, \ldots, M\}, m \in \mathcal{M}
$$

$\mathcal{C}$ : Set of inserting rescheduling period.

$$
\mathcal{C}=\{1, \ldots, C\}, c \in \mathcal{C}
$$

$\mathcal{W}$ : Set of complete rescheduling period.

$$
\mathcal{W}=\{1, \ldots, W\}, w \in \mathcal{W}
$$

$\mathcal{T}$ : Set of total rescheduling period that contains inserting and complete rescheduling periods.

$$
\mathcal{T}=\{1, \ldots, T\}, t \in \mathcal{T}
$$

\subsubsection{Parameters}

$C_{\mathrm{m}}$ : Capacity of the $m$-th equipment;

$D_{\mathrm{m}}$ : Full bucket factor of the $m$-th equipment;

$S_{\mathrm{i}}$ : Ore dispersion of the $i$-th stope;

$Y P_{\mathrm{t}}$ : Grade of the original in the $t$-th rescheduling period;

Yore $_{\mathrm{t}}$ : Total amount of ore mined in the $t$-th rescheduling period;

$p_{\mathrm{i}}$ : Grade of the $i$-th stope;

$z_{\mathrm{mij}}$ : The loaded travel time of $m$-th equipment from $i$-th stope to $j$-th ore pass;

$q_{\mathrm{mji}}$ : The unloaded travel time of $m$-th equipment from $i$-th stope to $j$-th ore pass;

$T_{\mathrm{c}}$ : Track of the $c$-th inserting rescheduling period;

$T_{\mathrm{w}}$ : Track of the $w$-th complete rescheduling period.

\subsubsection{Variables}

$n_{\text {cmij }}$ : The number of trips of $m$-th equipment from $i$-th stope to $j$-th ore pass in the $c$ th inserting rescheduling period;

$n_{\mathrm{cmji}}$ : The number of trips of $m$-th equipment from $j$-th ore pass to $i$-th stope in the $c$ th inserting rescheduling period;

$v_{\mathrm{ci}}$ : The amount of ore hauled from the $i$-th stope in the original planning of the $c$-th inserting rescheduling period;

$u_{\mathrm{cj}}$ : The amount of ore to be hauled to the $j$-th ore pass in the original planning of the $c$-th inserting rescheduling period;

$n_{\text {wmij: }}$ : The number of trips of $m$-th equipment from $i$-th stope to $j$-th ore pass in the $w$-th complete rescheduling period;

$n_{\mathrm{wmji}}$ : The number of trips of $m$-th equipment from $j$-th ore pass to $i$-th stope in the $w$-th complete rescheduling period; 
$v_{\text {wi }}$ : The amount of ore that has not been hauled out of the $i$-th stope in the original daily planning during the $w$-th complete rescheduling period;

$u_{\mathrm{wj}}$ : The amount of ore hauled to the $j$-th ore pass that was not completed in the original daily planning during the $w$-th complete rescheduling period;

$n_{\mathrm{tmi}}$ : The number of trips that the $m$-th equipment hauled ore from the $i$-th stope in the $t$-th rescheduling period.

\subsubsection{Rescheduling plan model}

In the actual production process of underground mines, equipment breakdown has a significant impact on the execution of the production tasks as well as the ore grade in the original scheduling planning. Therefore, the maximum completion rate of the ore mining planning and the smallest ore grade fluctuation in each rescheduling period is set as desired goals. In addition, the dressing plant has high requirements for the ore grade. Therefore, during each rescheduling period, the main goal is to minimize the fluctuation of the ore grade. The maximum completion rate of the planned ore mining volume is set as the secondary goal.

(1) The main objective function is to minimize the fluctuation of the ore grade during each rescheduling period:

$$
F 1=\left|\left(\frac{\sum_{\mathcal{M}} \sum_{\jmath} n_{\mathrm{tmi}} C_{\mathrm{m}} D_{\mathrm{m}} S_{\mathrm{i}} p_{\mathrm{i}}}{\sum_{\mathcal{M}} \sum_{\jmath} n_{\mathrm{tmi}} C_{\mathrm{m}} D_{\mathrm{m}} S_{\mathrm{i}}}\right)-Y P_{\mathrm{t}}\right|
$$

(2) The secondary objective function is to maximize the completion rate of ore mining during each rescheduling period:

$$
F 2=\left(\sum_{\mathcal{M}} \sum_{\jmath} n_{\mathrm{tmi}} C_{\mathrm{m}} D_{\mathrm{m}} S_{\mathrm{i}}\right) / \text { Yore }_{\mathrm{t}}
$$

According to the intersection of the breakdown repair period on the timeline, the constraint conditions are divided into inserting and complete rescheduling plan preparation conditions.

1) Inserting rescheduling plan preparation conditions:

$$
\begin{gathered}
\sum_{\mathcal{M}} \sum_{\mathcal{J}} n_{\mathrm{cmij}} C_{\mathrm{m}} D_{\mathrm{m}} S_{\mathrm{i}} \leq v_{\mathrm{ci}}, \forall c, i \\
\sum_{\mathcal{M}} \sum_{\jmath} n_{\mathrm{cmij}} C_{\mathrm{m}} D_{\mathrm{m}} S_{\mathrm{i}} \leq u_{\mathrm{cj}}, \forall c, j \\
\sum_{\jmath} \sum_{\jmath}\left(n_{\mathrm{cmij}} z_{\mathrm{mij}}+n_{\mathrm{cmji}} q_{\mathrm{mji}}\right) \leq T_{\mathrm{c}}, \forall c, m
\end{gathered}
$$

2) Complete rescheduling plan preparation conditions:

$$
\begin{gathered}
\sum_{\mathcal{M}} \sum_{\jmath} n_{\mathrm{wmij}} C_{\mathrm{m}} D_{\mathrm{m}} S_{\mathrm{i}} \leq v_{\mathrm{wi}}, \forall c, i \\
\sum_{\mathcal{M}} \sum_{\jmath} n_{\mathrm{wmij}} C_{\mathrm{m}} D_{\mathrm{m}} S_{\mathrm{i}} \leq u_{\mathrm{wj}}, \forall c, j \\
\sum_{\mathcal{J}} \sum_{\mathcal{J}}\left(n_{\mathrm{wmij}} Z_{\mathrm{mij}}+n_{\mathrm{wmji}} q_{\mathrm{mji}}\right) \leq T_{\mathrm{w}}, \forall w, m
\end{gathered}
$$

The constraint shown in Eq. (3) determines that the tonnage of ore hauled out of each stope during a single inserting rescheduling period cannot be greater than the tonnage of ore hauled out during that period in the originally planned task. The constraint shown in Eq. (4) determines that the tonnage of ore hauled to each ore pass during a single inserting rescheduling period cannot be greater than the original daily scheduled task. The constraint shown in Eq. (5) restricts the schedule duration of each piece of equipment during a single inserting rescheduling period, i.e., it cannot be greater than the duration of this period. Constraint determined by Eq. (6) indicates that the tonnage of ore hauled out of each stope in a complete rescheduling period cannot be greater than the tonnage of ore that has not been hauled out of the stope in the original plan. The constraint shown in Eq. (7) determines that the tonnage of ore hauled to each ore pass in a complete rescheduling period cannot be greater than the tonnage of ore hauled to the ore pass that was not completed in the original daily plan. Finally, the constraint determined by Eq. (8) restricts the schedule duration of each piece of equipment in the complete rescheduling period, i.e., it cannot be greater than the duration of this period.

\section{Optimization algorithm}

The wolf colony algorithm is based on the intelligence of the wolf colony, thereby simulating the predatory behavior of the wolf colony and its prey distribution method. This method abstracts three intelligent behaviors: wandering, rushing, and sieging. The 
wolf generation rule of "the winner takes it all" as well as the wolf colony update mechanism of "only the strong survive" are employed. This algorithm is characterized by high solution accuracy, fast convergence speed, few control parameters, and strong robustness. However, its disadvantage of falling into a local optimum requires enhancing the global search ability and increasing the diversity of the population to find the optimal solution in the global scope.

The wolf colony algorithm divides the population of an artificial wolf colony into three types of wolves: head wolves, detective wolves, and fierce wolves. In the solution space, the artificial wolf with the best fitness is the head wolf. The best section of artificial wolves (apart from the head wolf) in the solution space is regarded as detection wolves. The remaining population is designated as the fierce wolves. The head wolf is responsible for the command and maintenance of the entire wolf colony. The detective wolves hunt within the possible range of their prey, make independent decisions based on the scent left by the prey, and always search in the direction with the strongest smell. The fierce wolves are responsible for sieging the prey when detective wolves find it on the trail.

\subsection{Algorithm design and improvement}

(1) Population initialization

The equipment rescheduling plan problem is a typical Non-deterministic Polynomial (NP) problem. When solving this type of problem, the chromosome encoding method in the genetic algorithm is preferred for describing the solution of the problem. Therefore, the chromosome encoding method is employed to initialize the wolf colony. The combination of multiple chromosomes represents a feasible solution to the problem. The chromosome length is determined according to the problem scale and can be adjusted.

Lowercase characters are used for stopes, while capital characters are used for ore passes. For example, $[\mathrm{aAbBcC}]$ represents the operating route of a scraper. Multiple chromosomes form a single individual in the wolf colony, thereby indicating the current location of an artificial wolf. The position of the head wolf represents the position of the prey. For equipment rescheduling planning problems, the operation route of the scraper can be coded in periods. Each chromosome represents the operation route of the scraper in a specific period. After determining the chromosome coding method, the initial population is randomly generated according to the constraint conditions.

(2) Rules for determining the head wolf

First, the calculation method of individual fitness value is determined. The optimization model for equipment rescheduling planning is to minimize the objective function. Therefore, the reciprocal of the objective function is taken as the fitness of the individual. In addition, multiple objective functions correspond to multiple fitness values:

$$
\begin{aligned}
& \text { fitness } 1=\max \left(\frac{1}{F 1}\right) \\
& \text { fitness } 2=\max (F 2)
\end{aligned}
$$

Since the objective function is not unique and primary as well as secondary goals exist, the first fitness value of each artificial wolf in the solution space is compared against the head wolf rules. If there are multiple artificial wolves with the optimal first fitness values, the second fitness value is compared. If multiple equal second fitness values are present, one artificial wolf is randomly selected as the head wolf among all artificial wolves with equal first and second fitness values. The head wolf does not execute three intelligent behaviors. Moreover, it directly enters the next iteration.

\section{(3) Wandering behavior}

The wandering behavior of detective wolves determines the global search ability of the wolf colony algorithm. With an increase in the number of detective wolves, the chance of finding the global optimal solution is also increased. All artificial wolves in the solution space are first sorted. Then, better artificial wolves with half the population size (apart from the head wolf) are considered detective wolves. First, prey concentration $Y_{\mathrm{i}}$ at the 
current position of the $i$-th detective wolf is calculated. If $Y_{\mathrm{i}}$ is higher than the prey concentration $Y_{\text {lead }}$ perceived by the head wolf, then $Y_{\text {lead }}=Y_{\mathrm{i}}$. The $i$-th detective wolf initiates the call by replacing the wolf. If $Y_{\mathrm{i}} \leq Y_{\text {lead }}$, then the $i$-th detective wolf takes one step forward in $h$ directions and records the perceived prey concentration after each step before returning to the original position.

The detective wolf can advance in $h$ directions by randomly selecting $h$ gene positions in all chromosomes of the individual detective wolf. The step length is set as two. In other words, each time detective wolves wander a single step forward, the number of genes that are exchanged between each side of the selected gene position is increased by one.

For example, the position update method of wandering forward in the $h$-th direction for two steps is:

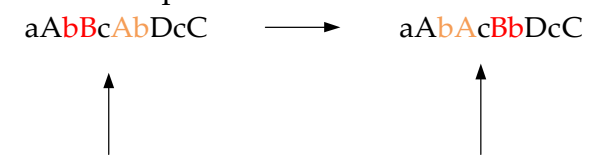

The $h$-th direction The $h$-th direction

To prevent the algorithm from falling into a local solution, when detective wolves wander in $h$ directions and are still in place after reaching the predetermined number of wandering, the detective wolves choose to move to a certain position in $h$ directions via the roulette method. Only fitness1 is selected in the roulette method.

(4) Rushing behavior

When detective wolves initiate a call, or the detective wolves fail to update the head wolf during the maximum number of wandering, the head wolf initiates a call. Once a call is initiated, all fierce wolves begin to rush towards the position of the head wolf. During that time, if the prey concentration $Y_{\mathrm{i}}$ perceived by the $i$-th fierce wolf is greater than $Y_{\text {lead }}$, then $Y_{\text {lead }}=Y_{\mathrm{i}}$. After all fierce wolves have completed rushing, the fierce wolf will transform into the head wolf and re-initiate a call. The rushing number is not calculated repeatedly. If $Y_{\mathrm{i}} \leq Y_{\text {lead }}$, the $i$-th fierce wolf continues to rush. When the maximum number of rushing is reached or the distance between the fierce wolf and the head wolf is less than $d$, the sieging behavior is started.

The rushing method of the fierce wolves is as follows. First, the rushing step length is set as two. Then, the gene exchange position $j$ of each chromosome of the $i$-th fierce wolf is randomly selected, and a single gene on each side of the gene position $j$ is exchanged with some position of the head wolf. The number of exchanged genes increases with the number of rushing.

The location update method for the second rushing of the $i$-th fierce wolf can be expressed as:

The $i$-th fierce wolf $\quad \mathrm{aAbBcAbDcC} \longrightarrow \mathrm{aAaAcCbDcC}$

The head wolf $\quad$ AAaAcCbDcC

The distance $d_{\mathrm{i}}$ between the fierce wolves and the head wolf can be calculated as:

$$
d_{i}=\mid \text { fitness } 1_{i}-\text { fitness } 1_{\text {lead }} \mid
$$

(5) Sieging behavior

When the fierce wolves get closer to the prey, they should unite with the detective wolf to conduct a close sieging on the prey and capture it. The position of the head wolf is regarded as the moving position of the prey.

The sieging method of artificial wolves is as follows. The sieging step is set to two. When each artificial wolf in the wolf colony participates in the sieging behavior, a gene position is first randomly determined. Then, the position of the artificial wolf is updated according to the location update method of the rushing behavior.

(6) "The winner takes it all" wolf generation rules 
The prey is distributed according to the principle of "from strong to weak", resulting in weak wolves being eliminated. To maintain the population diversity of the wolf colony, $40 \%$ of the artificial wolves with poor fitness are eliminated. Lastly, new artificial wolves are regenerated.

\subsection{Algorithm flow}

The specific operation flow of the algorithm is shown in Figure 2.

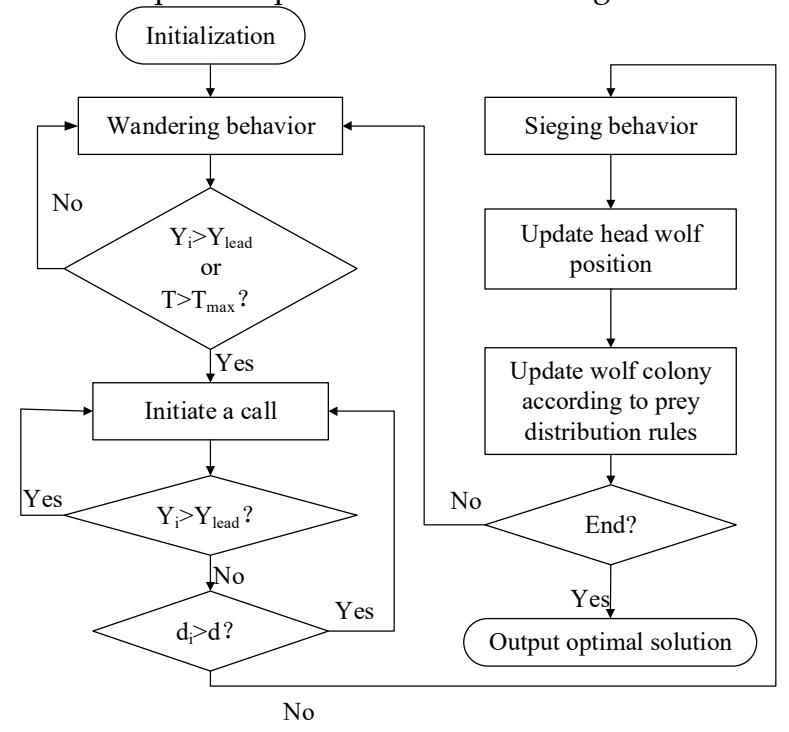

Figure 2. Improved wolf colony algorithm flow chart.

\section{Computational study}

\subsection{Basic data of the original scheduling plan}

The grades of the stopes currently being mined within a mine using the caving method are shown in Table 1. The loaded and empty running hours of the scraper between the stope and ore pass are shown in Tables 2 and 3. Part of the original scheduling plan tasks of each scraper on a certain day are shown in Table 4, in which lowercase characters indicate the number of stopes, and capital characters indicate the number of ore passes. During a certain production period, there are 7 stopes, 2 ore passes, and 3 scrapers in this sublevel. The equipment capacity of the scraper is $5 \mathrm{t}$, the full bucket coefficient is 0.95 , and the ore block dispersion is 0.83 . A simple demonstration of the running route of the $\# 1$ scraper at a certain time is shown in Figure 3.

Table 1. Grade table of each stope

\begin{tabular}{cc}
\hline Number of stopes & Grade/\% \\
\hline a & 24.03 \\
b & 61.74 \\
c & 44.26 \\
d & 36.46 \\
e & 49.15 \\
f & 49.28 \\
g & 56.74 \\
\hline
\end{tabular}

Table 2. Loaded travel time of the scraper between the stope and ore pass/s. 


\begin{tabular}{ccccccccc}
\hline & \multicolumn{7}{c}{ Stope/(Number) } \\
\cline { 2 - 8 } Pre & $\mathrm{a}$ & $\mathrm{b}$ & $\mathrm{c}$ & $\mathrm{d}$ & $\mathrm{e}$ & $\mathrm{f}$ & $\mathrm{g}$ \\
\cline { 2 - 8 } & $(1,1,4,3)$ & $(1,1,8,3)$ & $(1,1,11,3)$ & $(1,1,1,2)$ & $(1,1,6,2)$ & $(1,1,9,1)$ & $(1,1,12,1)$ \\
\hline A & 75.5 & 149.3 & 179.2 & 97.8 & 100.5 & 145.6 & 210.3 \\
B & 154.2 & 85.6 & 96.2 & 205.2 & 137.3 & 79.7 & 100.5 \\
\hline
\end{tabular}

Table 3. Empty travel time of the scraper between the stope and ore pass/s.

\begin{tabular}{cccccccc}
\hline & \multicolumn{7}{c}{ Stope/(Number) } \\
\cline { 2 - 8 } Ore & $\mathrm{a}$ & $\mathrm{b}$ & $\mathrm{c}$ & $\mathrm{d}$ & $\mathrm{e}$ & $\mathrm{f}$ & $\mathrm{g}$ \\
\cline { 2 - 8 } pass & $(1,1,4,3)$ & $(1,1,8,3)$ & $(1,1,11,3)$ & $(1,1,1,2)$ & $(1,1,6,2)$ & $(1,1,9,1)$ & $(1,1,12,1)$ \\
\hline $\mathrm{A}$ & 52.49 & 105.7 & 147.5 & 64.5 & 67.8 & 104.3 & 172.6 \\
$\mathrm{~B}$ & 110.7 & 57.5 & 61.3 & 144.2 & 84.6 & 54.0 & 68.2 \\
\hline
\end{tabular}

Table 4. Part of the operation route of the scraper.

\begin{tabular}{cc}
\hline Number & Operation route \\
\hline 1 & ......cAdBaBdAeAeBaAfBaBeAfBfAcBaBdAfBgAfAdBgBbBcAb...... \\
2 & .....BfAbBfBaBfBaAcAgBdAgAaBaAeAdBfAcAcAfAgBgAfAfBdBaAaAbA...... \\
3 & ......AeAcAfAcAgAfAdBcAdBeBaBgAcBdAgAgAfBdBbAfAgBfAbAeB...... \\
\hline
\end{tabular}

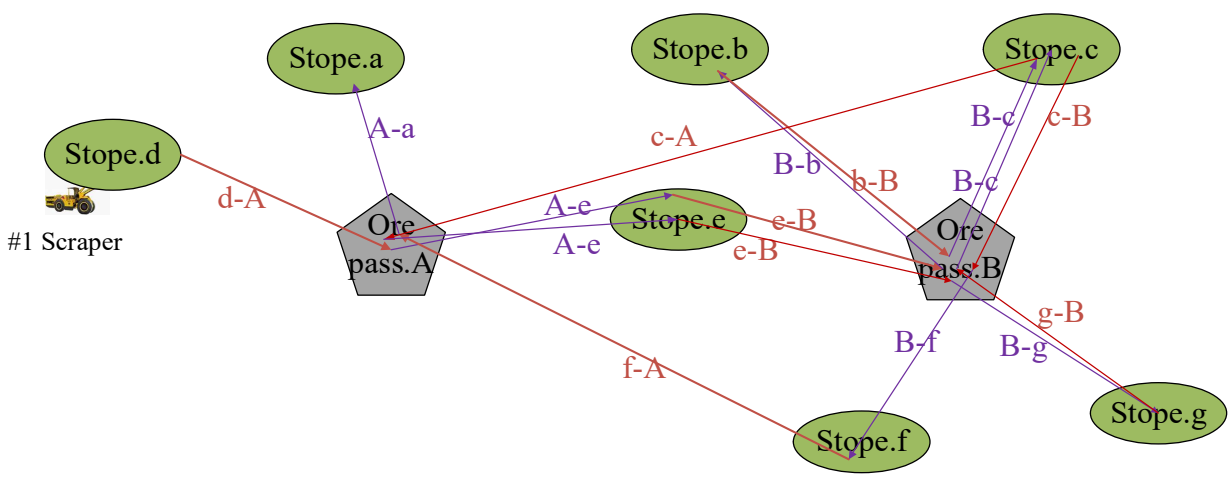

Figure 3. Operation route of \#1 scraper during a certain period.

The tonnage of daily ore mined in each stope, the grade of daily ore removal, and the tonnage of ore hauled to ore passes can be calculated according to each scraper's plans (Table 5, 6).

Table 5. The tonnage of ore mined in each stope and the grade of daily ore removal.

\begin{tabular}{ccc}
\hline Number of the stope & The tonnage of ore mined/t & $\begin{array}{c}\text { Grade of daily ore } \\
\text { removal/\% }\end{array}$ \\
\hline a & 512.53 & \\
b & 461.27 & \\
c & 595.32 & 45.94 \\
d & 646.57 & \\
e & 544.07 & \\
\hline f & 642.63 &
\end{tabular}


Number of the stope

The tonnage of ore mined/t

Grade of daily ore removal $/ \%$
g
666.28

Table 6. The tonnage of ore hauled to each ore pass.

\begin{tabular}{cc}
\hline Number of ore pass & The tonnage of ore hauled to ore pass/t \\
\hline A & 2310.31 \\
B & 1758.35 \\
\hline
\end{tabular}

\subsection{Equipment breakdowns simulation analysis}

During the actual production process, the equipment cannot operate continuously, and the occurrence of breakdowns will lead to the inaccurate completion of the originally planned tasks. The time node of the equipment breakdown cannot be predicted. However, equipment breakdown must be considered when preparing the plan. Therefore, it is necessary to simulate the breakdown of the scraper during the operation according to the breakdown rate of the equipment. Then, based on the simulation results, the equipment rescheduling plan is prepared.

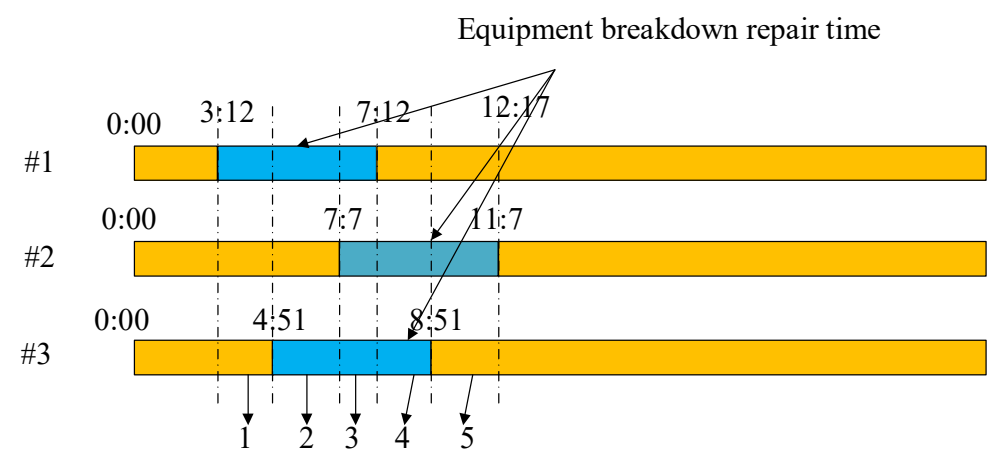

Figure 4. Analysis and demonstration of each period.

It is assumed that the change curve of the haulage equipment breakdown probability over time is $P_{\mathrm{tk}}=1-1.05^{-t}, t \geq 1$, where $t$ is an integer. Furthermore, it is assumed that the change curve can accurately simulate the occurrence of breakdowns, while the breakdown repair time is taken as four hours. The breakdown time of each piece of equipment, as well as the end time of breakdown maintenance, can be obtained through the random breakdown algorithm (Table 7).

Table 7. The simulated time of breakdown and maintenance end in each equipment.

\begin{tabular}{ccc}
\hline Number & Time of breakdown & $\begin{array}{c}\text { Time of breakdown maintenance } \\
\text { end }\end{array}$ \\
\hline 1 & $3: 12$ & $7: 12$ \\
2 & $7: 7$ & $11: 7$ \\
3 & $4: 51$ & $8: 51$ \\
\hline
\end{tabular}

The analysis of each period is shown in Figure 4, where periods 1, 2, 3, 4, and 5 are denoted as inserting rescheduling periods. When the rescheduling plan is prepared, each period is carried out in order. The next period can be prepared only after the rescheduling plan of the previous period is optimized. 


\subsection{Optimization results}

The number of original plan tasks in each period is taken as the constraint, while the ore grade deviation and the ore output in each period are taken as the target. Then, to compile the improved wolf colony algorithm code, the optimization plan of the rescheduling plan within each period is calculated via MATLAB software. In the wolf colony algorithm, the initial population size is 40 and the evolutionary algebra is 200 . The wandering step length, the rushing step length, and the sieging step length are all set as two. The distance threshold $d$ between the fierce wolf and the head wolf is based on the first fitness of the head wolf of each generation. This value can be set up to ten times lower than the first fitness value of the head wolf. When solving the planning model in each rescheduling period, the population has to be reinitialized and parameters have to be reset. The final solution results for each period are shown in Tables 8, 9, 10, and 11. During the third rescheduling period, all of the equipment is malfunctioning. Therefore, there is no need to prepare a rescheduling plan. To obtain a complete rescheduling plan for each piece of equipment, the solution results of each rescheduling period are inserted into the original plan. Then, the total tonnage of mined ore, the grade of daily ore removal, and the tonnage of ore hauled to each ore pass can be obtained (Table 12,13).

Table 8. The first rescheduling period plan optimization scheme.

\begin{tabular}{ccc}
\hline Number & Operation route & Fitness1 \\
\hline 2 & AgBgAdBeAcBdBdAfAfBgBfAcBbAfBfAg & \\
\cline { 2 - 2 } 3 & BgBfBbAeAfAaBdBeAfBeBeAb & $1.95 \mathrm{e}-3$ \\
& $\begin{array}{c}\text { BdAcAcAgBeAgBcAgAdAfAdBbBgAbAeBcA } \\
\text { bBeBgBgBfBeAeBbAa }\end{array}$ & \\
\hline
\end{tabular}

Table 9. The second rescheduling period plan optimization scheme.

\begin{tabular}{ccc}
\hline Number & Operation route & Fitness1 \\
\hline 2 & BcBbBaBbBcAbAfBcAeBfAaBdBgBcAeBfAfB & \multirow{2}{*}{ co75e-4 } \\
\hline
\end{tabular}

Table 10. The fourth rescheduling period plan optimization scheme.

\begin{tabular}{ccc}
\hline Number & Operation route & Fitness1 \\
\hline 1 & $\begin{array}{c}\text { cBgBbAbAcAbAcBaAcAfAeAgBbAcBcA } \\
\text { bBcBfBcAgBdAdAfBgAdBgAgBfBf }\end{array}$ & $4.45 \mathrm{e}-4$ \\
\hline
\end{tabular}

Table 11. The fifth rescheduling period plan optimization scheme.

\begin{tabular}{ccc}
\hline Number & Operation route & Fitness1 \\
\hline \multirow{2}{*}{1} & AfBfBfAeBcBeBeBfAgBbBgAgAfAgBfBaAbBaA & \\
& gAdBgBcBgAaBfAfAfAcBgAcBcBbBcAfAcAfAdA & \multirow{2}{*}{$1.51 \mathrm{e}-2$} \\
\cline { 2 - 2 } 3 & BcAcAfAfBfBcAbAgBgBgBbAbAeBeBbBgBdBdBd & \\
& BfAeAeBfBcBfAeAbAcAcAdAfBcBaBaBdB & \\
\hline
\end{tabular}

Table 12. The tonnage of ore mined in each stope and the grade of daily ore removal in the rescheduling plan. 
Number of the stope The tonnage of ore mined $/ t$

Grade of daily ore removal $/ \%$

\begin{tabular}{lll}
\hline a & 370.59 & \\
b & 417.91 & \\
c & 536.18 & 46.68 \\
d & 488.87 & \\
e & 441.56 & \\
f & 591.37 & \\
g & 571.66 \\
\hline
\end{tabular}

Table 13. The tonnage of ore hauled to each ore pass in the rescheduling plan.

\begin{tabular}{cc}
\hline Number of ore pass & The tonnage of ore hauled to ore pass/t \\
\hline A & 1864.80 \\
B & 1553.34 \\
\hline
\end{tabular}

\subsection{Result analysis}

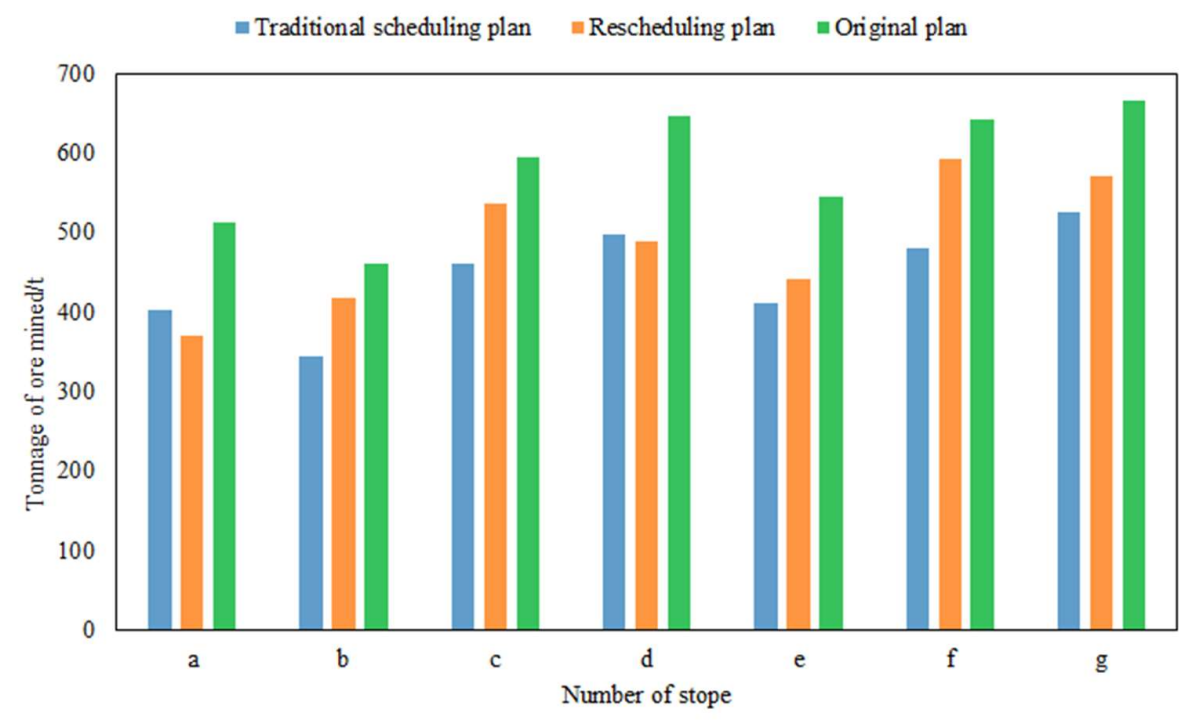

Figure 5. The amount of mined ore according to each scheme.

The rescheduling plan calculated by the optimization algorithm is compared with the traditional breakdown scheduling plan (Figure 5). The total ore tonnage of each stope in the rescheduling plan is higher than that of the traditional scheduling plan. It should be mentioned that the ore tonnage of some stopes in the rescheduling plan is lower than that of the traditional scheduling plan. According to Table 14, the tonnage of ore finally hauled to the ore pass in the rescheduling plan is approximately $300 \mathrm{t}$ higher than the ore tonnage of the traditional scheduling plan. Compared to the traditional scheduling plan, the rescheduling plan has a higher completion rate of $83.40 \%$, while the ore grade is increased by $0.74 \%$.

Table 14. Data comparison of various schemes. 


\begin{tabular}{cccc}
\hline Scheme & $\begin{array}{c}\text { The total tonnage of } \\
\text { ore mined/t }\end{array}$ & Plan completion rate/\% & $\begin{array}{c}\text { The grade fluctuation of } \\
\text { daily ore removal/\% }\end{array}$ \\
\hline Traditional scheduling plan & 3118.52 & 76.09 & 0 \\
Rescheduling plan & 3418.14 & 83.40 & 0.74 \\
\hline
\end{tabular}

\section{Discussion}

To successfully prepare the rescheduling plan and simplify the problem, several assumptions are made in this paper. However, due to many factors affecting the operation of the mining equipment, it is relatively difficult to predict its breakdowns. Therefore, accurately predicting the breakdown time of equipment will become one of the key aspects for accurate preparation of rescheduling plan as well as one of the important research directions in the future. In addition to the breakdown time prediction, the preparation of the equipment rescheduling plan conducted in this paper is a type of static preparation method. This type belongs to the category of short-term planning and can be used as preparation for subsequent research. However, the actual equipment operation plan is dynamic, i.e., it can be adjusted in real-time. Therefore, converting a static rescheduling plan into dynamic real-time breakdown dispatching represents an important research direction. In addition, when carrying out dynamic real-time breakdown dispatching of equipment, resources and environmental costs can be optimized as the targets. The aforementioned can be done to reduce environmental pollution and loss of natural resources, as well as to promote green mine production and construction.

\section{Conclusions}

In this paper, a model of the haulage equipment rescheduling plan based on the random simulation of equipment breakdowns was established. The model was optimized by improving the wolf colony algorithm. The following conclusions are made:

(1) According to the actual statistics of the number of haulage breakdowns in the mine, the breakdown probability can be calculated. Then, a random breakdown model, which is used to simulate the time of breakdown occurrence and maintenance end, is constructed by generating random numbers.

(2) When breakdown occurrence and maintenance end times are known, the breakdown period is divided into five inserting rescheduling periods by taking the crossing point between the breakdown time of each piece of equipment and the time when the breakdown occurs and maintenance ends. Then, during each rescheduling period, the rescheduling plan model is constructed based on random breakdown simulation. The main goal is to minimize the grade fluctuation in each period, while the secondary goal is to maximize the completion rate of the ore mining plan.

(3) The chromosome coding method of the genetic algorithm is used to improve the population individual form according to the wolf colony algorithm. The wandering step length, the rushing step length, the sieging step length, and the update method of the wolf colony individual position are refined to adapt to the solution of the rescheduling plan model. Finally, a wolf colony optimization algorithm is formed via string encoding. The algorithm has the characteristics of strong global search ability, fast convergence, and high robustness.

(4) The rescheduling plan optimized by the wolf colony algorithm is compared with the traditional scheduling planning. The results indicate that, when the equipment breaks down, the re-prepared scheduling plan has fewer fluctuations in the grade of daily ore removal (which is increased by $0.74 \%$ ). The completion rate is increased by $7.31 \%$ while the quality of the ore is simultaneously improved.

Author Contributions: Conceptualization, N.L.; Data curation, H.Y.; Formal analysis, S.F.; Funding acquisition, N.L., and M.J.; Investigation, Q.W. and T.L.; Methodology, N.L. and M.J.; Resources, 
H.Y., S.Z. and L.W.; Supervision, N.L.; Validation, S.F. and T.L.; Writing-original draft, S.F.; Writing-review \& editing, T.L., H.Y., Q.W., M.J., L.W.. All authors have read and agreed to the published version of the manuscript.

Funding: This research is supported by the National Key R\&D Program of China (Grant No. 2019YFC0605304).

Data Availability Statement: Not applicable.

Acknowledgments: Thanks to the relevant departments for funding and the mining companies that provided the experiment.

Conflicts of Interest: The authors declare no conflict of interest.

\section{References}

1. Yao, X., Hu, N., Zhou, L., Li, Y.. Ore blending of underground mines based on an immune clone selection optimization algorithm. Journal of University of Science and Technology Beijing. 2011, 33(05), 526-531.

2. Hou, J., Hu, N., Li, G., Ma, Z., Li, D., Gong, J.. Dynamic optimization of production plans for multi-metal underground mines. Chinese Journal of Engineering. 2016, 38(04), 453-460.

3. Campeau, L. P., Gamache, M.. Short-term planning optimization model for underground mines. Computers E Operations Research. 2016.

4. Li, G., Hou, J., Hu, N.. Integrated optimization model for production and equipment dispatching in underground mines. Chinese Journal of Engineering. 2018, 40(09), 1050-1057.

5. Yardimci, A. G., Karpuz, C.. Shortest path optimization of haul road design in underground mines using an evolutionary algorithm. Applied Soft Computing. 2019, 83.

6. Åstrand, M., Johansson, M., Zanarini, A.. Underground Mine Scheduling of Mobile Machines Using Constraint Programming and Large Neighborhood Search. Computers \& Operations Research. 2020, 123, 105036.

7. Samatemba, B., Zhang, L., Besa, B.. Evaluating and Optimizing the Effectiveness of Mining Equipment; the Case of Chibuluma South Underground Mine. Journal of Cleaner Production. 2020, 252, 119697.

8. M. Zandieh, M. Gholami. An immune algorithm for scheduling a hybrid flow shop with sequence-dependent setup times and machines with random breakdowns. International Journal of Production Research. 2009, 47(24), 6999-7027.

9. Al-Hinai, N., ElMekkawy, T. Y.. Robust and stable flexible job shop scheduling with random machine breakdowns using a hybrid genetic algorithm. International Journal of Production Economics. 2011, 132(2), 279-291.

10. Ai, X., Zhou, S., Chen Z., Zhao, Y.. Research on Optimal Scheduling Model and Solving Method for Power System with Interruptible Load Considering Multi Stochastic Factors. Proceedings of the CSEE. 2017, (08), 2231-2241.

11. Ba, Z., Yuan, Y., Dai, Y.. Robustness Measurement Approach of Job Scheduling with Machine Breakdowns. Computer Integrated Manufacturing Systems. 2020, 26(12), 3341-3349.

12. Peng, K., Pan Q., Gao, L., Li, X., Das, S., Zhang, B.. A Multi-Start Variable Neighbourhood Descent Algorithm for Hybrid Flowshop Rescheduling. Swarm and Evolutionary Computation. 2019, 45, 92-112.

13. Zhao, H., Galland, S., Knapen, L., Bellemans, L., Yasar, A. U. H.. Agent-Based Dynamic Rescheduling of Daily Activities. Procedia Computer Science. 2018, 130, 979-984.

14. Song, L., Gu, H., Jin, S., Zhao, H.. Rescheduling Methods for Manufacturing Firms Applying Make-to-Order Strategy. International Journal of Simulation Modelling. 2015, 14(4), 719-731.

15. Pang, X., Gao, L., Pan, Q.,Yu, S.. Intelligent Rescheduling System for Steelmaking and Continuous Casting Production. Proceedings of the 23rd International Conference on Industrial Engineering and Engineering Management 2016. Paris: Atlantis Press. 2017, $41-46$.

16. Gao, Y., Yang, L., Gao, Z.. Real-Time Automatic Rescheduling Strategy for an Urban Rail Line by Integrating the Information of Fault Handling. Transportation Research Part C: Emerging Technologies. 2017, 81, 246-267.

17. Moghaddam, S. K., Saitou, K.. On Optimal Dynamic Pegging in Rescheduling for New Order Arrival. Computers $\mathcal{E}$ Industrial Engineering. 2019, 136, 46-56.

18. Geng, Y., Yang, Y., Li B.. Rescheduling Optimization of Complex Mechanical Product Design Change Tasks. 2019 8th International Conference on Industrial Technology and Management (ICITM). 2019.

19. Li, Y., Carabelli, S., Fadda, E., Manerba, D., Tadei, R., Terzo, O.. Machine Learning and Optimization for Production Rescheduling in Industry 4.0. International Journal of Advanced Manufacturing Technology. 2020, 110(9-10), 2445-2463.

20. Gu, Z., Li, L., Zheng, J., Liu, G.. A Flexible Job-Shop Rescheduling Method by Considering the Machine Equipment Availability. Proceedings of the 28th Chinese Control and Decision Conference (2016 Ccdc). New York: Ieee. 2016, 4898-4902.

21. Wang, H., Wang, H., Li, Y., Wang, F.. Production Decision Rescheduling of Prefabricated Building Parts Subject to Interference from the Arrival of New Orders. International Journal of Industrial Engineering-Theory Applications and Practice. 2020, 27(5), 791-809.

22. Foroughi, S., Hamidi, J. K., Monjezi, M., Nehring, M.. The Integrated Optimization of Underground Stope Layout Designing and Production Scheduling Incorporating a Non-Dominated Sorting Genetic Algorithm (NSGA-II). Resources Policy. 2019, 63, 101408 . 
23. Wu, H., Xue, J., Xiao, R., Hu, J.. Uncertain Bilevel Knapsack Problem Based on An Improved Binary Wolf Pack Algorithm. Frontiers of Information Technology \& Electronic Engineering. 2020, 21(9), 1356-1368.

24. Zhou, X., Zhang, Q., Wang, T.. Intelligent Rescheduling Research on Train Dispatching Section of Main Line Highspeed Railway Based on Improved GWO Algorithm. 2020 IEEE 5th International Conference on Intelligent Transportation Engineering (ICITE). 2020. 


\section{MogoE迷 it}

\section{CERTIFICATE OF ENGLISH EDITING}

This is to certify that the manuscript entitled

\section{Rescheduling Plan Optimization of Underground Mine Haulage Equipment} Based on Random Breakdown Simulation

commissioned to us has been carefully edited by a native English-speaking editor of MogoEdit, and the grammar, spelling, and punctuation have been verified and corrected where needed. Based on this review, we believe that the language in this paper meets academic journal requirements. Please contact us with any questions.

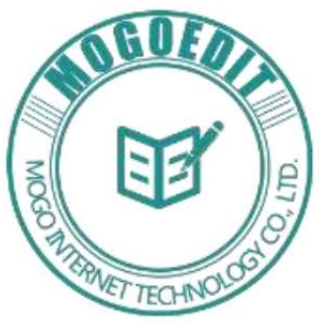

\section{Gang Zhang}

Dr. Gang Zhang Founder \& CEO of MogoEdit

Date of Issue December 22, 2021

Disclaimer: The changes in the document may be accepted or rejected by the authors in their sole discretion after our editing. However, MogoEdit is not responsible for revisions made to the document after our edit on December 22, 2021.

MogoEdit is a professional English editing company who provides English language editing, translation, and publication support services to individuals and corporate customers worldwide. As a company invested by the affiliate fund of Chinese Academy of Science, MogoEdit is one of the leading language editing service providers in China, whose clients come from more than 1000 universities and research institutes.

MogoEdit Website: http://en.mogoedit.com/

500+ native English editors: http://en.mogoedit.com/editors

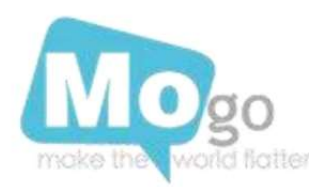

Mogo Internet Technology Co., LTD.

No. 57, 3rd Keji Road, Xi'an 710075, PR China +86 02988317483 support@mogoedit.com 